ARTICLE

Received 26 Feb 2014 | Accepted 20 Jun 2014 | Published 30 Jul $2014 \quad$ DOI: 10.1038/ncomms5470

\title{
Robust carbon dioxide reduction on molybdenum disulphide edges
}

\author{
Mohammad Asadi ${ }^{1}{ }^{\star}$, Bijandra Kumar ${ }^{1, \star}$, Amirhossein Behranginia', Brian A. Rosen ${ }^{2}$, Artem Baskin ${ }^{3}$, \\ Nikita Repnin ${ }^{3}$, Davide Pisasale ${ }^{1}$, Patrick Phillipss ${ }^{4}$, Wei Zhu ${ }^{5}$, Richard Haasch ${ }^{6}$, Robert F. Klie ${ }^{4}$, Petr Král ${ }^{3,4}$, \\ Jeremiah Abiade $^{1} \&$ Amin Salehi-Khojin ${ }^{1}$
}

Electrochemical reduction of carbon dioxide has been recognized as an efficient way to convert carbon dioxide to energy-rich products. Noble metals (for example, gold and silver) have been demonstrated to reduce carbon dioxide at moderate rates and low overpotentials. Nevertheless, the development of inexpensive systems with an efficient carbon dioxide reduction capability remains a challenge. Here we identify molybdenum disulphide as a promising cost-effective substitute for noble metal catalysts. We uncover that molybdenum disulphide shows superior carbon dioxide reduction performance compared with the noble metals with a high current density and low overpotential ( $54 \mathrm{mV}$ ) in an ionic liquid. Scanning transmission electron microscopy analysis and first principle modelling reveal that the molybdenum-terminated edges of molybdenum disulphide are mainly responsible for its catalytic performance due to their metallic character and a high $d$-electron density. This is further experimentally supported by the carbon dioxide reduction performance of vertically aligned molybdenum disulphide.

\footnotetext{
${ }^{1}$ Department of Mechanical and Industrial Engineering, University of Illinois at Chicago, Chicago, Illinois 60607, USA. ${ }^{2}$ Department of Chemical and Biomolecular Engineering, University of Illinois at Urbana-Champaign, Urbana, Illinois 61801, USA. ${ }^{3}$ Department of Chemistry, University of Illinois at Chicago, Chicago, Illinois 60607, USA. ${ }^{4}$ Department of Physics, University of Illinois at Chicago, Chicago, Illinois 60607, USA. ${ }^{5}$ Dioxide Materials, Champaign, Illinois 61820, USA. ${ }^{6}$ Materials Research Laboratory, University of Illinois at Urbana-Champaign, Urbana, Illinois 61801, USA. ${ }^{*}$ These authors contributed equally to this work. Correspondence and requests for materials should be addressed to A.S.-K. (email: salehikh@uic.edu).
} 
$\mathrm{D}$ uring the last few decades, the amount of carbon dioxide $\left(\mathrm{CO}_{2}\right)$ present in the environment has reached the highest level (396.80 p.p.m.) of the last 20 million years ${ }^{1}$, causing radical and largely unpredictable changes in the environment ${ }^{2-4}$. Recent efforts have revealed that $\mathrm{CO}_{2}$ can be converted by electrochemical reduction processes using renewable energy sources into energy-rich modules, offering an efficient path for both $\mathrm{CO}_{2}$ remediation and an alternative energy source ${ }^{5-9}$. However, the $\mathrm{CO}_{2}$ reduction by electrochemical processes faces various fundamental and practical challenges mainly due to highcost and unsatisfactory conversion performance of existing catalytic systems ${ }^{5}$. Numerous physical and chemical approaches have been employed to improve the performance of existing $\mathrm{CO}_{2}$ reduction systems $\mathrm{s}^{10-14}$ without achieving a major breakthrough.

Recently, molybdenum disulphide $\left(\mathrm{MoS}_{2}\right)$ and related layered materials have attracted a significant attention due to their low price and prominent catalytic features. $\mathrm{MoS}_{2}$ became widely used as an efficient catalyst for hydrodesulphurization ${ }^{15,16}$, oxygen reduction reactions ${ }^{17}$ and hydrogen evolution reaction $(\mathrm{HER})^{16,17}$. Moreover, it was demonstrated that $\mathrm{MoS}_{2}$ has a noteworthy performance for water splitting, approaching that of Pt-group metals ${ }^{17-19}$.

In this study, we report that layer-stacked bulk $\mathrm{MoS}_{2}$ with molybdenum (Mo)-terminated edges exhibits the highest $\mathrm{CO}_{2}$ reduction performance reported to date, to the best of our knowledge. This performance is demonstrated in a diluted solution of 1-ethyl-3-methylimidazolium tetrafluoroborate $\left(\mathrm{EMIM}-\mathrm{BF}_{4}\right)$ ionic liquid that is, $4 \mathrm{~mol} \% \mathrm{EMIM} \mathrm{BF}_{4}$ and $96 \mathrm{~mol} \%$ water. EMIM-BF 4 is deliberately selected due to its' particular catalytic features, which make the system more selective for carbon monoxide (CO) formation than hydrogen $\left(\mathrm{H}_{2}\right)$ production ${ }^{7-9}$. In the same diluted electrolyte, commonly used silver nanoparticles (Ag NPs) exhibit moderate performance, whereas a bulk silver $(\mathrm{Ag})$ catalyst is unable to reduce $\mathrm{CO}_{2}$. The high catalytic activity of bulk $\mathrm{MoS}_{2}$ is attributed to the Mo-terminated edges, where the Mo atoms possess approximately one order of magnitude higher ( $d$ orbital) electronic density than $\mathrm{Ag}$ atoms at the surface of an $\mathrm{Ag}$ film, as shown by our first principle calculations. The lower work function $(3.9 \mathrm{eV})$ also promotes the advanced performance of the $\mathrm{MoS}_{2}$ catalyst ${ }^{20}$. The performance of the $\mathrm{MoS}_{2}$ catalyst is further improved by designing an atomic edge-terminated surface via synthesizing vertically aligned $\mathrm{MoS}_{2}$.

\section{Results}

Characterization. Figure 1a and b shows optical and scanning electron microscopy images, respectively, of the layered structure $^{21,22}$ of our bulk $\mathrm{MoS}_{2}$ sample (Supplementary Fig. 1). Such layered assemblies offer a large number of edges (inset of Fig. 1b), which are believed to be highly electrocatalytically active sites in electrochemical reactions ${ }^{19,23}$. To further detail the atomic arrangement, scanning transmission electron microscopy (STEM) analysis was performed on several mechanically exfoliated, mono- and multi-layer-thick sheets of $\mathrm{MoS}_{2}$ flakes. Since the STEM high-angle annular dark-field (HAADF) image intensity relies on the atomic number $(Z)$, it delivers direct information about the arrangement of Mo and $\mathrm{S}$ atoms in the a

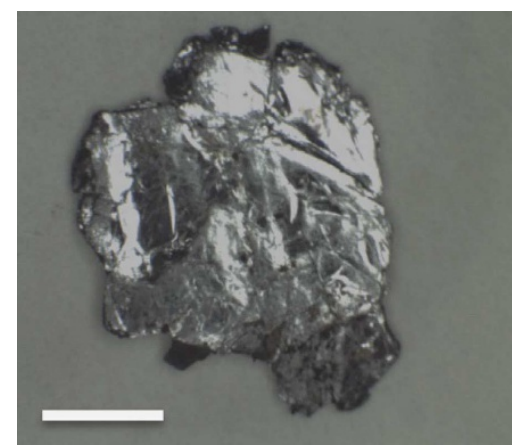

d

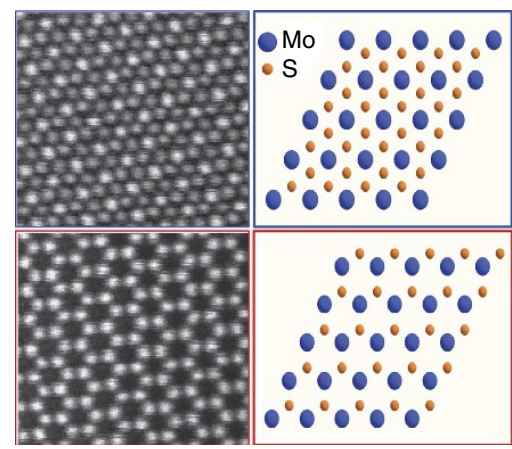

b

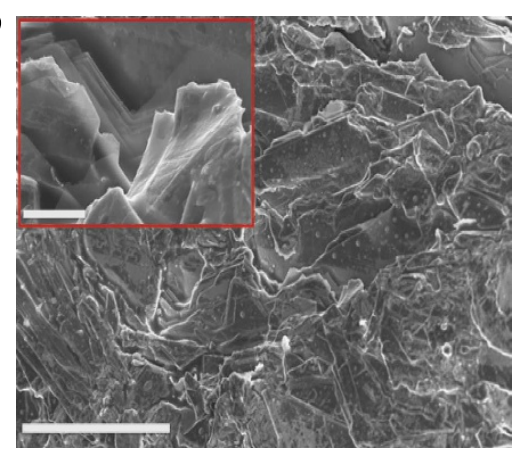

e

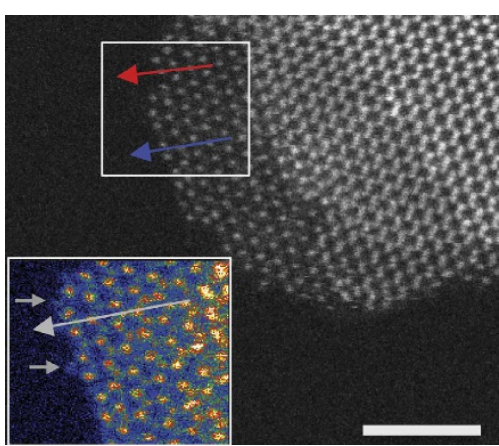

C

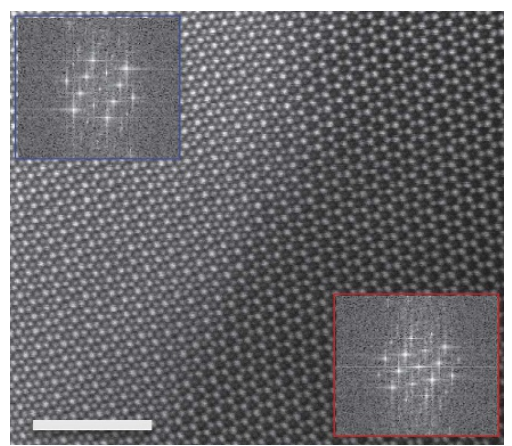

$\mathbf{f}$

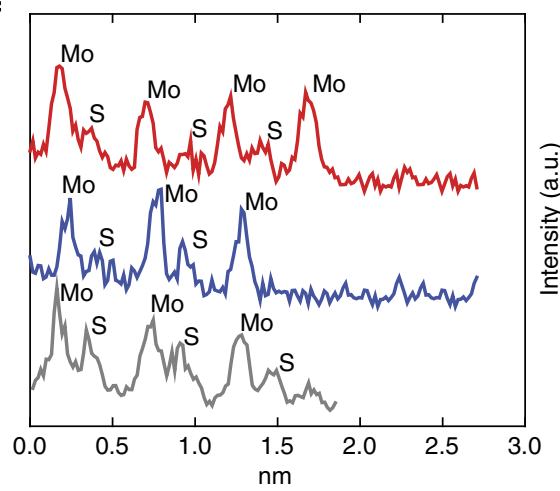

Figure 1 | Structural and elemental analysis of $\mathbf{M o S}_{\mathbf{2}}$. (a) Optical image of bulk MoS 2 used as catalyst (scale bar, 2 mm), (b) Scanning electron microscopy images of the $\mathrm{MoS}_{2}$ displaying the stacked-layered structure and sharp edges of the $\mathrm{MoS}_{2}$ flakes. Scale bars are 50 and $5 \mu \mathrm{m}$ (for inset), respectively, and (c) high-angle annular dark-field (HAADF) images (scale bar, $5 \mathrm{~nm}$ ) showing both the $1 \mathrm{~T}$ (blue) and $2 \mathrm{H}$ (red) phases of MoS 2 , along with their respective Fast Fourier Transforms (FFTs) (inset). (d) Higher magnification HAADF images show clearly distinct atomic configuration corresponding to the $1 \mathrm{~T}$ (top) and $2 \mathrm{H}$ (bottom) type of $\mathrm{MoS}_{2}$. The related schematic atomic models have also been shown on the right side. (e) Raw greyscale HAADF and false-color low-angle annular dark-field (LAADF) image (inset) of $\mathrm{MoS}_{2}$ edges (scale bar, $5 \mathrm{~nm}$ ) and (f) the line scans (red and blue towards edges) identifying Mo atoms to be the terminating atoms in the general case. In limited instances, an additional light atom (grey line scan) occupying what should be a Mo-position, most probably a carbon atom, from the STEM substrate. 
$\mathrm{MoS}_{2}$ film. The results of the STEM structural (Fig. 1c) and Fast Fourier transform analyses (Supplementary Fig. 2) show that the $\mathrm{MoS}_{2}$ layers are made of two clearly distinct structural domains consisting of $1 \mathrm{~T}$ (octahedral) and $2 \mathrm{H}$ (triangular prismatic). The magnified images (atomic resolution) of selected regions confirm the coexistence of both $1 \mathrm{~T}$ and $2 \mathrm{H}$ atomic arrangements (Fig. 1d).

Identification of the atoms on the $\mathrm{MoS}_{2}$ edges is also crucially important, as the Mo and $\mathrm{S}$ atoms possess entirely different electronic structures. Figure 1e shows the edge of a $\mathrm{MoS}_{2}$ flake imaged in HAADF and low-angle annular dark-field (LAADF) (inset) mode. The line intensity profiles (plotted towards vacuum) suggest that the edges of the $\mathrm{MoS}_{2}$ flakes are Mo terminated (Fig. 1f). This finding is in agreement with the earlier work by Zhou et. al. ${ }^{24}$ who report that the Mo-terminated edges have the lowest formation energy in free-standing single-layer $\mathrm{MoS}_{2}$. In rare instances, a substitutional defect (atom) appears at the $\mathrm{MoS}_{2}$ edge. Based on the LAADF image (inset of Fig. 1e) and the line intensity profile (grey line), it is clear that this is a lighter atom (compared with S), most likely a carbon atom (from the underlying holey carbon STEM grid). Hence, the STEM analysis undoubtedly validates the presence of Mo atoms on the edges of $\mathrm{MoS}_{2}$ flakes.

Catalytic evaluation. The $\mathrm{CO}_{2}$ reduction ability of bulk $\mathrm{MoS}_{2}$ covered by flakes with exposed Mo-terminated edges was first

a

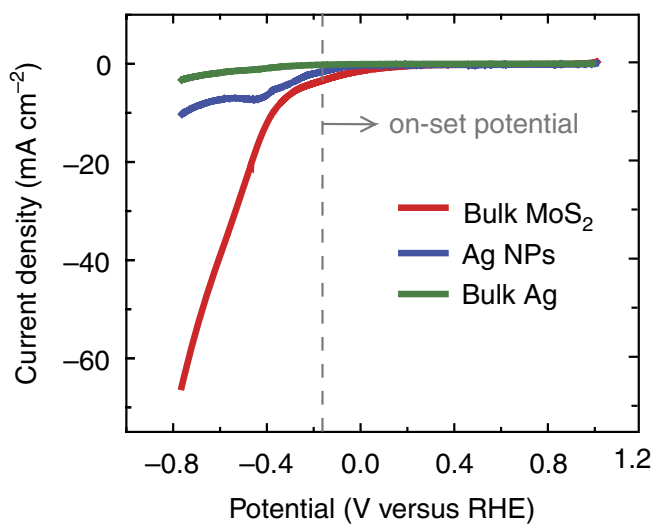

C

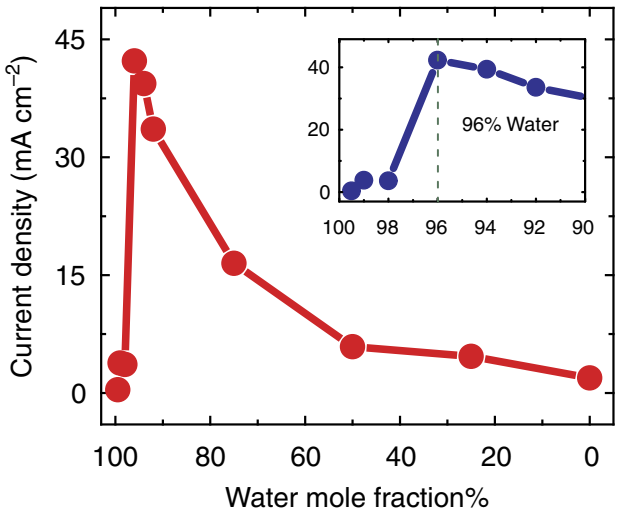

examined by performing cyclic voltammetry (CV). The applied voltage was swept between +1.0 and $-0.764 \mathrm{~V}$ versus reversible hydrogen electrode (RHE; in the present study, all potentials are reported with respect to RHE) with a $15 \mathrm{mV} \mathrm{s}^{-1}$ scan rate. The experiments were conducted in a two-compartment three-electrode electrochemical cell (Supplementary Fig. 3) using argon (Ar) or $\mathrm{CO}_{2}$-saturated $96 \mathrm{~mol} \%$ water-4 mol\% EMIM-BF solution $(\mathrm{pH} \sim 4)$ as an electrolyte. Figure $2 \mathrm{a}$ represents the $\mathrm{CV}$ curve for the $\mathrm{CO}_{2}$ reduction. It should be noted that the $\mathrm{CO}_{2}$ reduction equilibrium potential is $-0.11 \mathrm{~V}$ versus RHE in the protic media ${ }^{7,10}$. We observed that $\mathrm{CO}_{2}$ reduction reaction initiates at $-0.164 \mathrm{~V}$ confirmed by measuring $\mathrm{CO}$ as a product by gas chromatography (GC) system (CO Faradaic efficiency $\mathrm{FE}=\sim 3 \%)$. This suggests a very low overpotential $(54 \mathrm{mV})$ for $\mathrm{CO}$ formation in our system. At $-0.2 \mathrm{~V}(90 \mathrm{mV}$ overpotential), approximately 7\% CO formation FE was measured (Fig. 2b). $\mathrm{MoS}_{2}$ also exhibits a significantly high $\mathrm{CO}_{2}$ reduction current density $\left(65 \mathrm{~mA} \mathrm{~cm}^{-2}\right.$ at $\left.-0.764 \mathrm{~V}\right)$, where $\mathrm{CO}_{2}$ is selectively converted to $\mathrm{CO}(\mathrm{FE} \sim 98 \%)$. However, at the same potential $(-0.764 \mathrm{~V})$, the bulk $\mathrm{Ag}$ catalyst shows a considerably lower current density $\left(3 \mathrm{~mA} \mathrm{~cm}^{-2}\right.$ ) (Fig. 2a) but for the $\mathrm{H}_{2}$ formation (Supplementary Fig. 4a). Ag NPs (average diameter of $40 \mathrm{~nm}$ ) show only a current density of $10 \mathrm{~mA} \mathrm{~cm}^{-2}$ with $65 \%$ selectivity for the $\mathrm{CO}$ formation under the same experimental conditions (Fig. 2a and Supplementary Fig. 4b). In addition, the $\mathrm{CO}_{2}$ reduction current density for $\mathrm{MoS}_{2}$ is also significantly higher

b

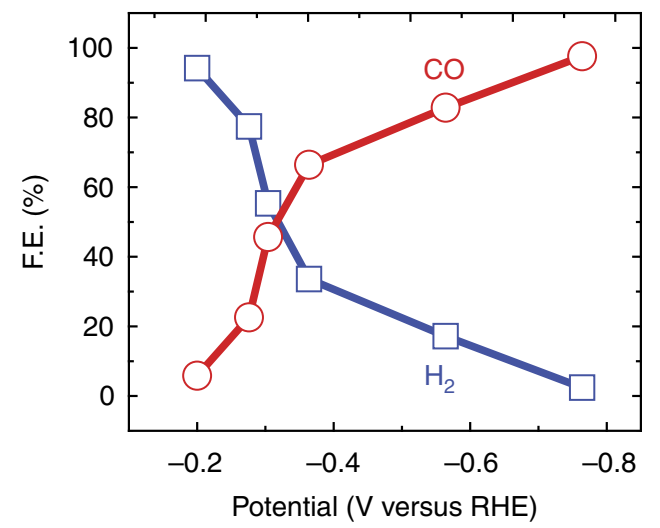

d

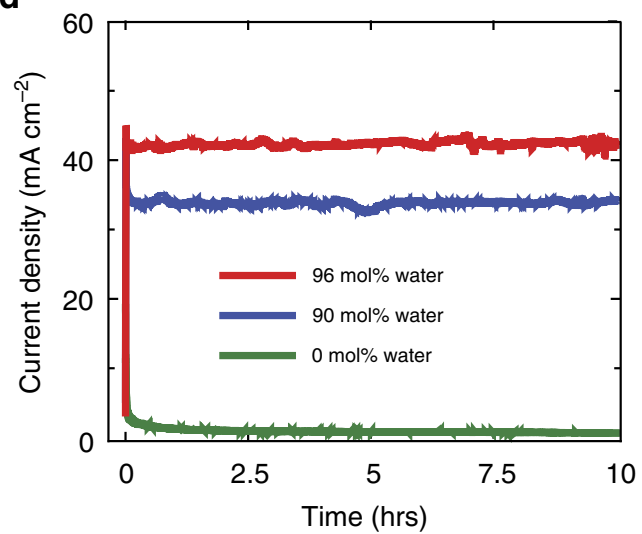

Figure 2 | $\mathbf{C O}_{\mathbf{2}}$ reduction performance of the bulk $\mathbf{M o S}_{\mathbf{2}}$ catalyst in the $\mathbf{E M I M}^{-B_{\mathbf{4}}}$ solution. (a) Cyclic voltammetric (CV) curves for bulk MoS ${ }_{2}, \mathrm{Ag}$

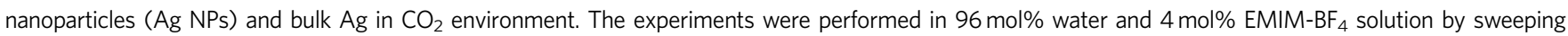
applied potential from $+1 \mathrm{~V}$ to $-0.764 \mathrm{~V}$ versus RHE. The vertical grey line indicates the low overpotential $(\sim 54 \mathrm{mV})$ for $\mathrm{CO}_{2}$ reduction at bulk MoS 2 . (b) $\mathrm{CO}$ and $\mathrm{H}_{2}$ Faradaic efficiency (FE) at different applied potentials. (c) The current density of $\mathrm{CO}_{2}$ reduction (measured by Chrono-Amperometry) at $0.764 \mathrm{~V}$

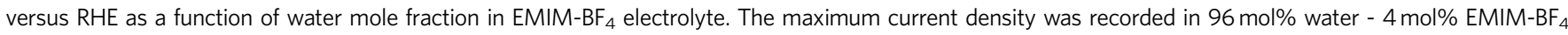
electrolyte (inset). The $\mathrm{pH}$ value of the solutions was also monitored (Supplementary Table 1). (d) Chrono-Amperometry results of $\mathrm{MoS}_{2}$ catalyst in different solutions (96, 90 and 0 mol\% water) showing negligible loss in current density even after $10 \mathrm{~h}$. 
than the maximum current density $\left(\sim 8.0 \mathrm{~mA} \mathrm{~cm}^{-2}\right)$ achieved when Ag NPs were used in the dynamic electrochemical flow cell using a similar electrolyte solution ${ }^{13}$. For all the cases, the current densities were normalized against the geometrical surface area $9,10,16-17,25-26$. Surprisingly, the $\mathrm{MoS}_{2}$ catalyst also shows a high current density $\left(50 \mathrm{~mA} \mathrm{~cm}^{-2}\right)$ in an Ar-saturated electrolyte, where only $\mathrm{H}_{2}$ was detected as the major product (Supplementary Note 1 and Supplementary Fig. 5). These results are discussed further in supporting information.

Figure $2 \mathrm{~b}$ shows the measured $\mathrm{FE}$ of the $\mathrm{CO}$ and $\mathrm{H}_{2}$ formation for a wide range of applied potentials between -0.2 and $-0.764 \mathrm{~V}$. Interestingly, depending on the applied potential, $\mathrm{MoS}_{2}$ effectively operates as a catalyst for both $\mathrm{CO}_{2}$ reduction and HER. $\mathrm{CO}_{2}$ is converted at $\mathrm{MoS}_{2}$ into a tunable mixture of $\mathrm{H}_{2}$ and CO (syngas), ranging in each component from 0 to $\sim 100 \%$. The variation in $\mathrm{FE}$ of $\mathrm{CO}$ and $\mathrm{H}_{2}$ as a function of the applied potential originates from the differences in the $\mathrm{CO}_{2}$ and HER reduction mechanisms. In principle, the favourable thermodynamic potential for the $\mathrm{H}_{2}$ evolution is lower than $\mathrm{CO}_{2}$ reduction. As the applied potential exceeds the onset potential of the $\mathrm{CO}_{2}$ reduction $(-0.164 \mathrm{~V})$, this reaction is activated. Essentially, two $\mathrm{H}^{+}$are consumed for a $\mathrm{CO}$ formation as a result of one $\mathrm{CO}_{2}$ molecule reduction ${ }^{10,13,27-29}$. Thus, a fraction of both the existing $\mathrm{H}^{+}$(from the electrolyte) and the electrons (on the catalyst surface) are consumed in $\mathrm{CO}_{2}$ reduction reactions instead of HER reactions. In addition, the EMIM- $\mathrm{CO}_{2}$ complex works as an inhibitor for the $\mathrm{H}_{2}$ formation in $\mathrm{HER}^{7}$.

Here, it is useful to compare the $\mathrm{MoS}_{2}$ catalyst performance with the existing results for noble metal catalysts (Fig. 3 and Supplementary Fig. 6). It should be noted that current density represents the $\mathrm{CO}$ formation rate, whereas $\mathrm{FE}$ shows the amount of current density consumed to produce $\mathrm{CO}$ during the $\mathrm{CO}_{2}$ reduction reaction. Thus, we compared the catalysts' overall performance by multiplying these two parameters at different overpotentials. Interestingly, we noticed that bulk $\mathrm{MoS}_{2}$ exhibits the highest performance at all overpotentials. At low overpotentials $(0.1 \mathrm{~V})$, bulk $\mathrm{MoS}_{2}$ shows almost 25 times higher $\mathrm{CO}_{2}$ reduction performance compared with the $\mathrm{Au} \mathrm{NPs}{ }^{10}$ and $\sim 1.3$ times higher than the Ag NPs. At higher overpotentials $(0.4 \mathrm{~V})$, bulk $\mathrm{MoS}_{2}$ exhibits approximately one order of magnitude higher performance than Ag NPs and more than two times higher than recently reported nanoporous $\mathrm{Ag}^{14}$. At this overpotential, the $\mathrm{Au}$ NPs compete with bulk $\mathrm{MoS}_{2}$. It should be noted that $\mathrm{MoS}_{2}$ produce $\mathrm{H}_{2}$ as a by-product which allows obtaining directly synthetic gas, whereas $\mathrm{Au}$ NPs produces formic acid $\left(\mathrm{HCOO}^{-}\right)$ as a by-product in the examined conditions ${ }^{10}$. As mentioned previously, bulk $\mathrm{Ag}$ is unable to reduce $\mathrm{CO}_{2}$ in the examined experimental conditions. Moreover, the $\mathrm{Cu}$ performance $\mathrm{e}^{11}$ remains below that of Ag NPs, Au NPs and bulk $\mathrm{MoS}_{2}$. These results clearly indicate that $\mathrm{MoS}_{2}$ exhibits the highest $\mathrm{CO}_{2}$ reduction performance reported so far.

Next, we investigated how the water mole fraction affects the catalytic activity of the $\mathrm{MoS}_{2}$ catalyst for the $\mathrm{CO}_{2}$ reduction (Fig. 2c). We observe that the $\mathrm{CO}_{2}$ reduction current density largely grows above $90 \mathrm{~mol} \%$ water solution densities (inset Fig. 2c) and reaches a maximum in the $96 \mathrm{~mol} \%$ water solution. The addition of water molecules can tailor the $\mathrm{pH}$ value (that is, $\mathrm{H}^{+}$concentration) of the electrolyte ${ }^{13,30,31}$ (Supplementary Table 1) and consequently affect the electrochemical reduction reaction rate. The $\mathrm{pH}$ of the electrolyte fluctuates due to the hydrolysis of $\mathrm{BF}_{4}^{-}$, which produces anions (for example, $\left.\left(\mathrm{BF}_{3} \mathrm{OH}\right)^{-}\right)$and $\mathrm{HF}^{13,30,31}$. It should be noted that the overall $\mathrm{CO}_{2}$-to-CO conversion reaction requires both electrons and protons ${ }^{13}$. Our density functional theory (DFT) calculations show significantly higher density (more than one order of magnitude) of $d$ electrons on Mo-edge atoms compared with Ag (for details, see DFT section), suggesting that the concentration of protons $\left(\mathrm{H}^{+}\right)$is the rate-determining part of the $\mathrm{CO}_{2}$ reduction reaction. Thus, the attained maximum rate of the reduction process is attributed to: (i) the high concentration of $\mathrm{H}^{+}(\mathrm{pH} \sim 4)$ in the reaction media and (ii) the low viscosity of the solution. It is clear that the low viscosity allows for a high diffusion rate of the reactants (EMIM- $\mathrm{CO}_{2}^{-}$and $\mathrm{H}^{+}$) towards the catalyst's active edge sites ${ }^{27,32-34}$. A similar trend was observed for Ag NPs catalysts in a dynamic electrochemical flow cell when the maximum current density $\left(\sim 8 \mathrm{~mA} \mathrm{~cm}^{-2}\right)$ was obtained in a $90 \mathrm{~mol} \%$ water electrolyte ${ }^{13}$

Additionally, a catalyst's stability is a major issue to be addressed. Thus, we examined the stability of the catalyst for a prolonged period $(10 \mathrm{~h})$ in 96,90 and $0 \mathrm{~mol} \%$ water solutions. As

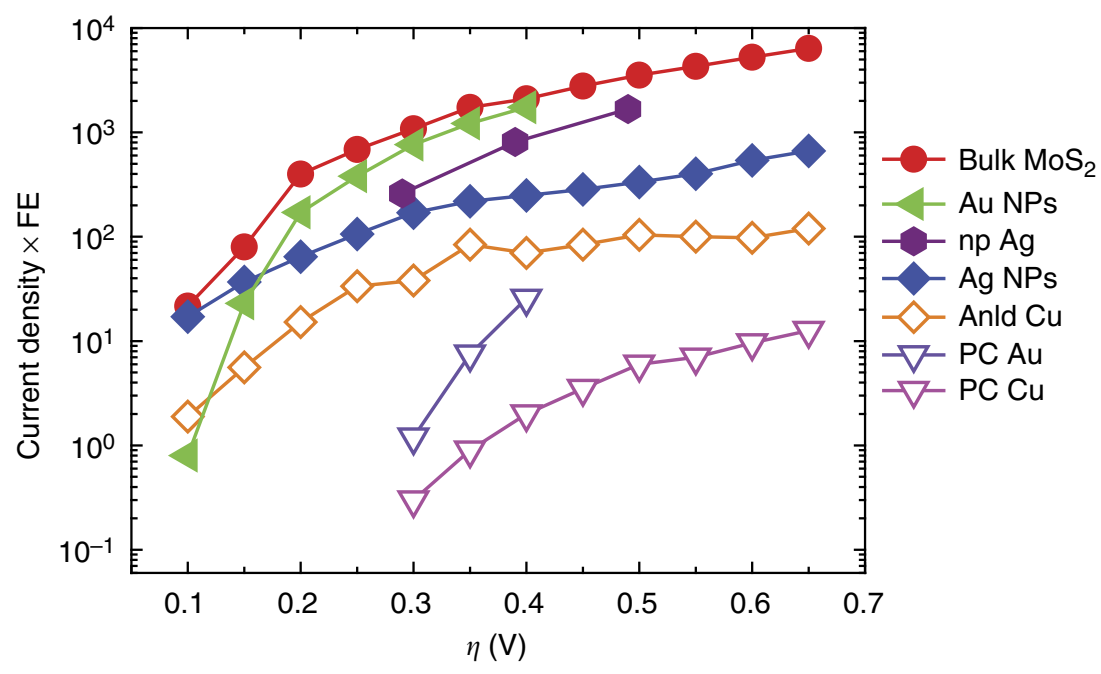

Figure 3 | Overview of different catalysts' performance at different overpotentials. Bulk $\mathrm{MoS}_{2}, \mathrm{Ag}$ nanoparticles (Ag NPs) and bulk $\mathrm{Ag}$ results were taken from the present study where electrochemical experiments were performed in similar conditions. Oxidized Au nanoparticles (Au NPs) and polycrystalline $\mathrm{Au}\left(\mathrm{PC} \mathrm{Au}\right.$ ) data were carefully extracted from the reference ${ }^{10}$. Annealed copper ( $\mathrm{Anld} \mathrm{Cu}$ ) and polycrystalline $\mathrm{Cu}(\mathrm{PC} \mathrm{Cu}$ ) data were taken from reference ${ }^{11}$. Nanoporous $\mathrm{Ag}\left(\mathrm{np} \mathrm{Ag}\right.$ ) data were extracted from reference ${ }^{14} \cdot \mathrm{CO}_{2}$ reduction performance curve of bulk $\mathrm{Ag}$ is not shown as its performance is almost zero in experimental conditions discussed in the text. The extended results are provided in the supporting file. 
a

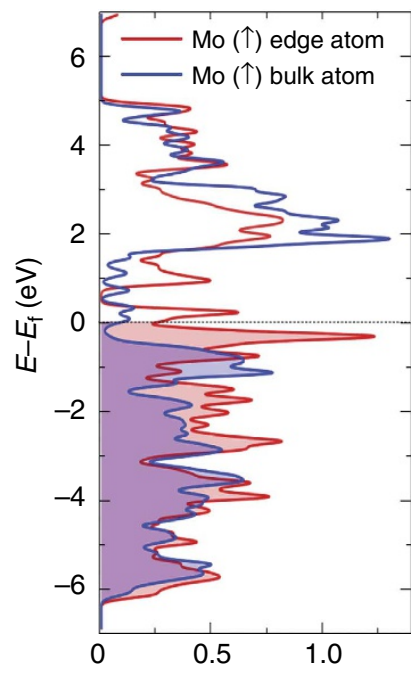

b

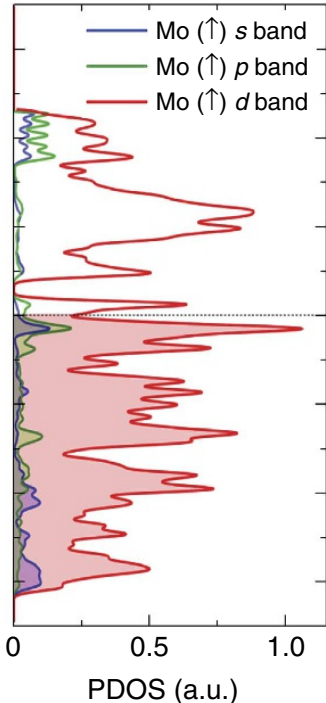

c

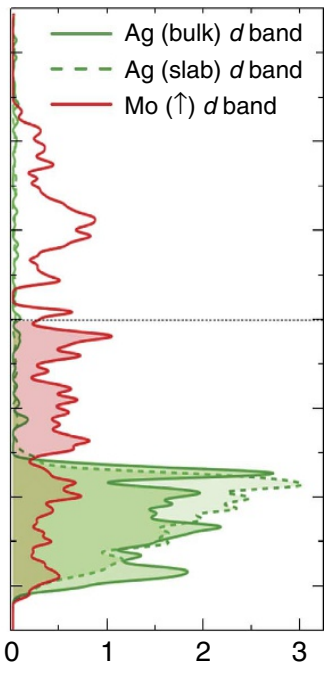

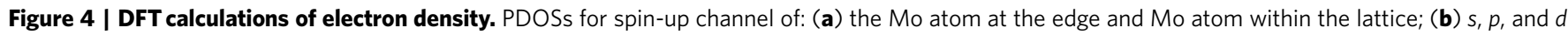
orbital of Mo-edge atom. (c) PDOS of $d$ band of Mo-edge atom, Ag atom from bulk and Ag-slab of 8.32 - $\AA$ thickness. Electron density on Mo-edge atom is significantly ( $\sim 11$ times) higher than the electron density on $\mathrm{Ag}$ atom.

seen in Fig. 2d, the steady-state current densities remain stable for the studied time $(10 \mathrm{~h})$, providing evidence of the long-term stability and efficiency of the $\mathrm{MoS}_{2}$ catalyst.

\section{Discussion}

To elucidate the origin of the high $\mathrm{CO}_{2}$ reduction rate on the $\mathrm{MoS}_{2}$ catalyst, we calculated its projected density of states (PDOS) per different Mo and S atoms ${ }^{20,35,36}$, using DFT methods (for computational details, see method section). The density of states at the Fermi energy level $\left(E_{\mathrm{f}}\right)$ roughly determines the availability of electrons for a given reaction ${ }^{35}$. We found that the electronic structure of $\mathrm{MoS}_{2}$ ribbons (Supplementary Note 2) is near $E_{\mathrm{f}}$ formed by edge bands of only one-spin polarization, originating from the Mo and $\mathrm{S}$ atoms exposed at both $\mathrm{MoS}_{2}$ edges. In the vicinity of $E_{\mathrm{f}}$, the spin-polarized PDOS for these Mo atoms is approximately twice larger than that of the bulk Mo atoms (Fig. 4a). Since the bulk Mo atoms, sandwiched between two $S$ layers, are not directly exposed to the electrolyte, the $\mathrm{MoS}_{2}$ catalytic activity should be primarily related to the edge states formed by Mo-edge atoms (Supplementary Fig. 7). The $S$ atoms possess less reactive $p$ orbitals (Supplementary Fig. 8), and they are not present at the catalytically active edge sites (confirmed by STEM).

Next, we resolved the PDOS of the Mo-edge atoms into $s^{-}, p_{-}$ and $d$-orbital electron contributions (Fig. $4 \mathrm{~b}$ ). The obtained data indicate that near $E_{\mathrm{f}}$, the PDOS is dominated by $d$-orbital (Mo) electron states, which are known to actively participate in catalysed reactions ${ }^{35}$. The Mo $d$ electrons form metallic edge states $^{37}$ (Supplementary Note 2), which can freely supply electrons to the reactants attached at the edges. To assess how the Mo-edge states are affected by the presence of additional $\mathrm{MoS}_{2}$ layers, we performed the same analysis for a double-layer $\mathrm{MoS}_{2}$ strip. Our calculations showed that an interlayer coupling further increases the $d$-electron PDOS near $E_{\mathrm{f}}$ (Supplementary Note 2 and Supplementary Fig. 9a-d). In the presence of an external bias, all these $d$-electron states near $E_{\mathrm{f}}$ can be accessed in the reaction, supporting the large observed $\mathrm{MoS}_{2}$ activity. Finally, we compared our $d$-orbital PDOS in Mo-edge atoms with that in $\mathrm{Ag}$ atoms in two structures: a bulk Ag and a two-dimensional slab
Ag (both fcc lattice with a lattice constant of $4.09 \AA$ ) of a $8.32-\AA$ thickness (after relaxation) (Fig. 4c). We found that the $d$-band centre for Mo-edge atoms is closer to the Fermi energy level than that in both $\mathrm{Ag}$ structures. This can partly explain the high catalytic activity of $\mathrm{MoS}_{2}$, since the higher the $d$-band centre is, the more reactive the metal is due to a lower transition state energy ${ }^{35}$. Moreover, the PDOS of Mo-edge atoms near $E_{\mathrm{f}}$ is approximately one order of magnitude higher than the PDOS of Ag atoms, suggesting the availability of the excess of $d$ electrons on the Mo-edge atoms. We believe that both these factors are mainly responsible for the high $\mathrm{CO}_{2}$ reduction current density of $\mathrm{MoS}_{2}$.

To reveal the role of EMIM ions in carrying $\mathrm{CO}_{2}$ molecules, we have also performed quantum molecular dynamics simulations (TeraChem) of the $[\text { EMIM-CO }]^{+}$complex hydrated in quantum water. We have tested the effect of different $\mathrm{pH}$ of the solution on the $\left[\mathrm{EMIM}-\mathrm{CO}_{2}\right]^{+}$complex stability in several possible configurations. Our simulations reveal that $\mathrm{CO}_{2}$ most likely binds to $\mathrm{EMIM}^{+}$through the $\mathrm{C} 4 / 5$ protons than through the $\mathrm{C} 2$ proton (known to provide stronger binding in vacuum). In this configuration, the complex appears more stable (bond length) and it also provides a better protection against the conversion of $\mathrm{CO}_{2}$ into $\mathrm{HCO}_{3}^{-}$and $\mathrm{CO}_{3}^{2-}$ species.

Our simulations revealed that the $\mathrm{EMIM}^{+}$cation forms a complex [EMIM-CO $]^{+}$with $\mathrm{CO}_{2}$ stabilized by $\mathrm{H}_{2}$ bonding (Supplementary Note 3 and Supplementary Fig. 10). However, the complex form depends on the $\mathrm{pH}$ of the electrolyte. In neutral solution, within $\sim 2 \mathrm{ps}$, the $\left[\mathrm{EMIM}-\mathrm{CO}_{2}\right]^{+}$complex reacts with water molecule, forming either the $\left[\mathrm{EMIM}-\mathrm{HCO}_{3}\right]$ or $[\mathrm{EMIM}-$ $\left.\mathrm{CO}_{3}\right]^{-}$complexes (Supplementary Fig. 10a). It is well known ${ }^{38}$ that in neutral and basic conditions, $\mathrm{HCO}_{3}^{-}$and $\mathrm{CO}_{3}^{2-}$ are the dominant species, respectively. However, our quantum molecular dynamics simulations reveal that in acidic environment, similar to our experimental conditions $(\mathrm{pH}<4)$, the $\left[\mathrm{EMIM}-\mathrm{CO}_{2}\right]^{+}$ complex remains stable (Supplementary Fig. 10).

These results agree with our previous in situ EMIM- $\mathrm{CO}_{2}$ complex formation studies ${ }^{8}$. The $\left[\mathrm{EMIM}-\mathrm{CO}_{2}\right]^{+}$complexes could potentially physisorb (Coulombic and van der Waals coupling) ${ }^{7-9}$ at the (negatively charged) $\mathrm{MoS}_{2}$ cathode, resulting in a close encounter of the $\mathrm{CO}_{2}$ molecules with the $\mathrm{MoS}_{2}$ surface. 
a

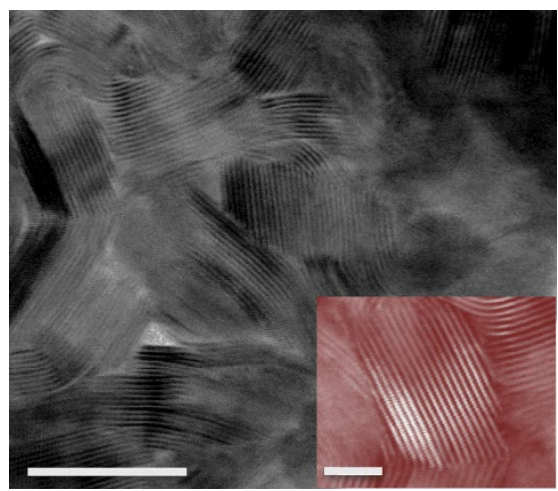

C

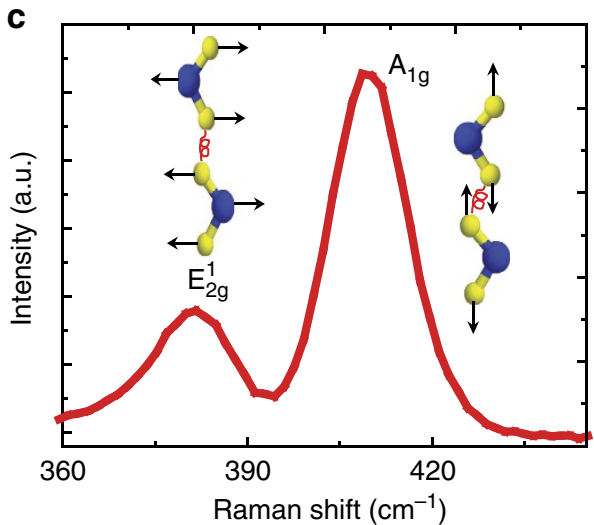

b

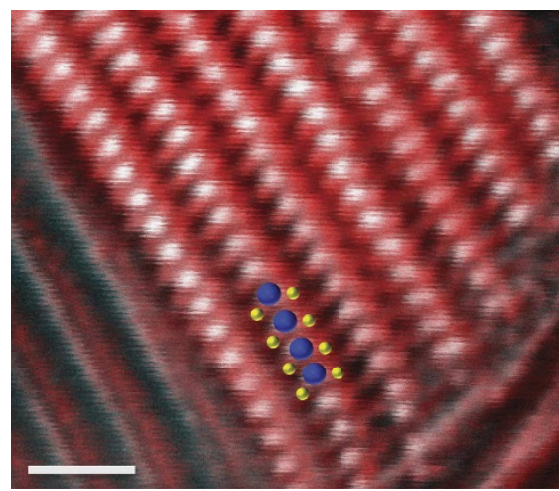

d

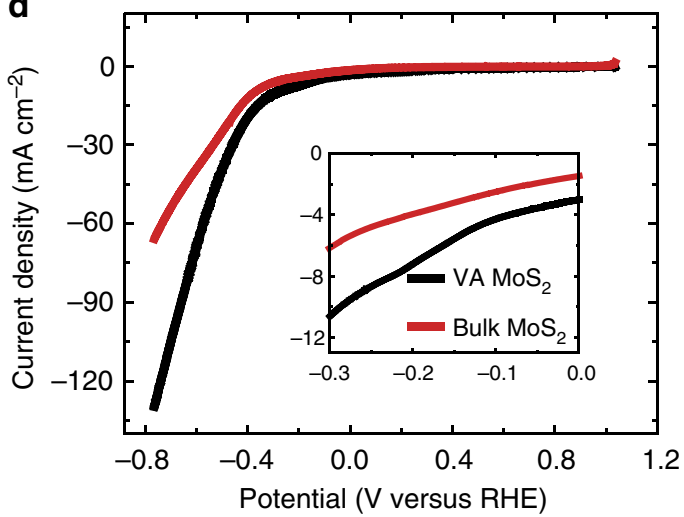

Figure 5 | Vertically aligned $\mathbf{M o S}_{\mathbf{2}}$ nanoflakes. (a) Annular bright-field (ABF) STEM images of vertically aligned MoS 2 (scale bar, $20 \mathrm{~nm}$ ). STEM analysis (inset) shows the vertically aligned texture of $\mathrm{MoS}_{2}$ nanoflakes (scale bar, $5 \mathrm{~nm}$ ). (b) RGB added image of ( $\mathrm{G}+\mathrm{B}$ ) high-angle annular dark-field (HAADF) (R) inverted ABF STEM images of vertically aligned $\mathrm{MoS}_{2}$. High-resolution HAADF STEM image of vertically aligned MoS 2 (scale bar, $2 \mathrm{~nm}$ ). Mo atoms are brighter and larger in size in comparison with sulphur atoms due to high atomic number. (c) Raman spectrum for vertically aligned $\mathrm{MoS}_{2}$. (d) $\mathrm{CO}_{2}$ reduction performance of bulk $\mathrm{MoS}_{2}$ and vertically aligned $\mathrm{MoS}_{2}$ represented by VA MoS .

The presence of $\mathrm{EMIM}^{+}$cations around $\mathrm{CO}_{2}$ molecules can reduce the reaction barrier for electrons passing into $\mathrm{CO}_{2}$. Thus, the observed high- $\mathrm{CO}_{2}$ reduction reaction is attributed to a synergistic action of the $\mathrm{MoS}_{2}$ catalyst and the EMIM-BF $\mathrm{B}_{4}$ ionic liquid. While EMIM- $\mathrm{BF}_{4}$ has a crucial role by reducing the overpotential for the reaction, the $\mathrm{CO}_{2}$ reduction rate is mainly governed by the intrinsic properties of the $\mathrm{MoS}_{2}$ catalyst. In addition, we measured the work function of $\mathrm{MoS}_{2}$ through the use of ultraviolet photoelectron spectroscopy. The obtained results indicate that the work function of $\mathrm{MoS}_{2}(3.9 \mathrm{eV})$ is significantly lower than that of the bulk $\mathrm{Ag}(4.37 \mathrm{eV})$ and $\mathrm{Ag}$ NPs $(4.38 \mathrm{eV})^{9}$. Due to the low work function of $\mathrm{MoS}_{2}$, the abundant metallic-like $d$ electrons in its edge states can take part in the reactions, ultimately resulting in the superior $\mathrm{CO}_{2}$ reduction performance compared with Ag.

Now that we understand that the $\mathrm{MoS}_{2}$ edges are responsible for the high $\mathrm{CO}_{2}$ reduction reaction rate, we have synthesized a vertically aligned $\mathrm{MoS}_{2}$ nanosheet ${ }^{39}$, and observed another factor of two improvements on the $\mathrm{CO}_{2}$ reduction performance. In brief, a 5-nm thick layer of Mo was deposited on glassy carbon substrate by electron beam evaporation, followed by sulphurization by exposing the film to a sulphur vapour stream at $700^{\circ} \mathrm{C}$. Figure $5 \mathrm{a}$ presents a HAADF and annular bright-field image of the vertically aligned $\mathrm{MoS}_{2}$ nanosheets. While the $\mathrm{MoS}_{2}$ layers are generally aligned perpendicular to the substrate surface, only a few select sheets can be found which are aligned parallel to the electron beam to allow for atomic resolution imaging (Fig. 5b). This image identifies the clearly separated Mo and $S$ atomic columns, as the Mo atoms are heavier and thus appear brighter. The proposed atomic structure of the Mo and S layers is superimposed on the atomic resolution image in Fig. 5b. While the nature of the terminating atoms in these $\mathrm{MoS}_{2}$ nanosheets cannot be directly visualized in this orientation, previous results have shown that synthesized $\mathrm{MoS}_{2}$ nanosheets are generally terminated by Mo atoms due to their low-energy state ${ }^{24}$. The vertically aligned $\mathrm{MoS}_{2}$ samples were further characterized by Raman spectroscopy (Fig. 5c). Two essential peaks are clearly visible at $385 \mathrm{~cm}^{-1}$ (in-plane Mo-S phonon mode $-E^{1}{ }_{2 \mathrm{~g}}$ mode) and $408 \mathrm{~cm}^{-1}$ (out-of-plane Mo-S phonon mode $-A^{1} \mathrm{~g}$ mode), respectively ${ }^{21,25,39,40}$. The ratio of out-ofplain $A^{1}$ phonon mode to $E_{2 \mathrm{~g}}^{1}$ mode is significantly high $(\sim 3)$, which clearly supports the existence of vertically orientated nature of $\mathrm{MoS}_{2}$ flakes ${ }^{39}$.

Figure $5 \mathrm{~d}$ shows the $\mathrm{CO}_{2}$ reduction performance of the vertically aligned $\mathrm{MoS}_{2}$ obtained in similar experimental conditions (that is, $96 \mathrm{~mol} \%$ water and $4 \mathrm{~mol} \%$ EMIM-BF 4 ). As expected, $\mathrm{CO}_{2}$ reduction reaction initiated at low overpotential $(54 \mathrm{mV})$ similar to bulk $\mathrm{MoS}_{2}$. Additionally, further improvement has been observed within complete applied potential range (Fig. 5d). In the low-applied potential region, vertically aligned $\mathrm{MoS}_{2}$ exhibits two times higher $\mathrm{CO}_{2}$ reduction current density compared with the bulk $\mathrm{MoS}_{2}$ as shown in inset of Fig. 5d. This trend remains also valid in the high-potential region. At $-0.764 \mathrm{~V}$, a remarkably high $\mathrm{CO}_{2}$ reduction current density $\left(130 \mathrm{~mA} \mathrm{~cm}^{-2}\right)$ was recorded for vertically aligned $\mathrm{MoS}_{2}$. The high catalytic performance of vertically aligned $\mathrm{MoS}_{2}$ is attributed to the high density of active sites preferably Mo atoms available for the $\mathrm{CO}_{2}$ reduction reaction. However, further efforts are 
needed to understand the nature of edge atoms in the vertically aligned $\mathrm{MoS}_{2}$ responsible for $\mathrm{CO}_{2}$ reduction performance.

In summary, we have discovered that bulk $\mathrm{MoS}_{2}-\mathrm{a}$ nonprecious catalyst and the most versatile member of TMDCsexhibits the highest observed catalytic performance for the $\mathrm{CO}_{2}$ reduction. The significantly higher $\mathrm{CO}_{2}$ reduction current density (relative to noble metal catalysts) is mainly attributed to a high density of $d$ electrons in Mo-terminated edges and also to its low work function. We propose that TMDCs can successfully replace expensive noble metal electrodes, with the promise of higher $\mathrm{CO}_{2}$ conversion rates and selectivity and can be the most attractive catalyst for future $\mathrm{CO}_{2}$ reduction applications.

\section{Methods}

$\mathbf{M o S}_{\mathbf{2}}$ characterization. Morphology of $\mathrm{MoS}_{2}$ was visualized at different scales. Optical characterizations were performed by using a Stereo-F $(\times 16-\times 100$ microscope) at $\times 2$ magnification and digital images of bulk $\mathrm{MoS}_{2}$ (purchased through SPI Supplies) were taken using a 5 mega pixels charge-coupled device camera mounted on the microscope. Scanning electron microscopy was performed to characterize the morphology of the bulk $\mathrm{MoS}_{2}$ at microscale. The instrument used for characterization is integrated in a Raith e-LiNE plus ultra-high-resolution electron beam lithography system. During imaging, the samples were kept at a distance of $10 \mathrm{~mm}$ from the electrons source and the voltage was kept at $10 \mathrm{kV}$. No particular types of preparation were implemented before imaging. To visualize atomic structure, STEM was performed using a probe-corrected JEOL JEMARM200CF equipped with a $200 \mathrm{kV}$ cold-field emission gun. Images were acquired in either the H/LAADF, with the former providing an approximately $Z^{2}$ contrast, while the latter is more sensitive to lower angle scattering. A 14 m.r.a.d. probe convergence angle was used for imaging, with the HAADF and LAADF detector angles set to 54-220 and 24-96 m.r.a.d., respectively. Annular bright-field images were also collected to identify $S$ atomic columns, as annular bright-field excels in the imaging of light elements; a collection angle of 7-14 m.r.a.d. was used. For STEM experiments, $\mathrm{MoS}_{2}$ flakes obtained by mechanical exfoliation of bulk $\mathrm{MoS}_{2}$ (standard Scotch-tape method) were directly transferred on QUANTIFOIL R 2/1 Holey films with $2-\mu \mathrm{m}$ circular holes by copper grid (200 mesh, purchased from the Electron Microscopy Sciences). The intensity line profile was attained by using Gatan Digital Micrograph. Both the Web Electron Microscopy Applications Software (WebEMAPS) and CrystalMaker Software programs were also employed to generate and visualize the crystal structures schematically.

Raman spectroscopy. Raman spectroscopy (Renishaw Raman 2000) was used to detect the $\mathrm{MoS}_{2}$ in-plane and out-of-plane phonon mode. The spectrum was obtained by exposing small pieces of the samples that is, bulk $\mathrm{MoS}_{2}$ (without any particular treatment) to $514-\mathrm{nm}$ laser beam (Ar laser, power $10 \mathrm{~mW}$ and spot size $10 \mu \mathrm{m})$.

Ultraviolet photoelectron spectroscopy. Surface work function measurements were carried out using ultraviolet photoelectron spectroscopy (UPS). UPS data were acquired with a Physical Electronics PHI 5400 photoelectron spectrometer using He I $(21.2 \mathrm{eV})$ ultraviolet radiation and a pass energy of $8.95 \mathrm{eV}$. To separate the signal arising from secondary electron emission from the detector to that from the secondary electron emission from the sample, a -9 -V bias was applied to the sample using a battery.

Electrochemical experiments. To examine the catalytic activity of $\mathrm{MoS}_{2}$ for $\mathrm{CO}_{2}$ reduction, electrochemical experiments were carried out in a custom made two-compartment three-electrode electrochemical cell (Supplementary Fig. 3). The compartments were separated by a physical barrier using glass frit. Bulk $\mathrm{MoS}_{2}$ (purchased through SPI Supplies), platinum (Pt) gauze 52 mesh (purchased via Alfa Aesar) and Ag wire (annealed 99.9\% metal basis, purchased from Alfa Aesar) were used as working, counter and reference electrode, respectively. EMIM-BF was purchased through Sigma-Aldrich. Electrolytes with different water mole fractions were prepared by adding known volume of deionized water into EMIM-BF 4 . Electrochemical $\mathrm{CO}_{2}$ reduction experiments were performed in anaerobic $\mathrm{CO}_{2}$ (AirGas)-saturated electrolyte. The applied voltage was swept between +1.0 and $-0.764 \mathrm{~V}$ versus RHE with a $15 \mathrm{mV} \mathrm{s}^{-1}$ scan rate. Cyclic voltammetry (CV) curves were then recorded using a Voltalab PGZ100 potentiostat (purchased via Radiometer Analytical SAS) calibrated with a RCB200 resistor capacitor box. The potentiostat was connected to a PC using Volta Master (version 4) software. For chrono-Amperometry measurement, $\mathrm{CO}_{2}$ concentration was kept constant with bubbling high-purity $\mathrm{CO}_{2}$ in solution along with mixing during experiment. Current densities were normalized with catalyst geometrical surface area ${ }^{10,16,17,25-26}$.
Product analysis. Electrochemical experimental yields were analysed by GC in SRI $8610 \mathrm{C}$ GC system equipped with $72 \times 1 / 8$-inch S.S. molecular sieve-packed column and a thermal conductivity detector. Production of $\mathrm{CO}$ and $\mathrm{H}_{2}$ was examined separately. Ultra high-purity helium (purchased through AirGas) was used as a carrier gas for $\mathrm{CO}$ detection, whereas ultra high-purity nitrogen (AirGas) was utilized for $\mathrm{H}_{2}$ detection (Supplementary Note 4). Initially, GC system was calibrated for $\mathrm{CO}$ and $\mathrm{H}_{2}$. A JEOL GCMate II (JEOL USA, Peabody MA, USA) gas chromatograph/mass spectrometer was further used to prove that yielded $\mathrm{CO}$ is only $\mathrm{CO}_{2}$ electrochemical reduction product. The gas chromatograph was an Agilent 6890Plus (Wilmington DE) equipped with a G1513A auto-injector with 100 vial sample tray connected to a G1512A controller. The GC column was a fused silica capillary column with a nonpolar 5\% phenyl $95 \%$ dimethylpolysiloxane phase (Agilent HP-5ms Ultra Inert), 30 meters long, $0.25 \mathrm{~mm}$ internal diameter, $0.25 \mu \mathrm{m}$ film thickness.

To confirm that the $\mathrm{CO}$ product is derived from $\mathrm{CO}_{2}$, an isotope $13 \mathrm{CO}_{2}$ was used as feedstock and GC-Mass spectroscopy was used for gas detection. Mass spectrometer was a bench top magnetic sector operating at a nominal resolving power of 500 using an accelerating voltage of $2,500 \mathrm{v}$. The spectrometer was operated in full scan EI mode ( + ve) with the filament operating at $70 \mathrm{eV}$ scanning from $\mathrm{m} / z 10$ to $\mathrm{m} / \mathrm{z} 400$ using a linear magnet scan. The scan speed was $0.2 \mathrm{~s}$ per scan. Data analysis was performed using the TSSPro software (Shrader Analytical \& Consulting Laboratories, Detroit MI, USA) provided with the spectrometer. Mass calibration was performed using perflourokerosene.The results are discussed in Supplementary Note 5 and Supplementary Fig. 11.

Synthesize of vertically aligned $\mathbf{M o S}_{\mathbf{2}}$. Vertically aligned $\mathrm{MoS}_{2}$ nanoflakes were grown by chemical vapour deposition using a slightly modified method as reported previously ${ }^{39}$. At first, substrates (glassy carbon) were thoroughly cleaned via rinsing in acetone, methanol and isopropanol solvents sequentially followed by drying in nitrogen flow. Next, a thin layer of Mo $(5 \mathrm{~nm})$ was deposited on the substrates by electron beam evaporation (Varian Evaporation System). For sulphurization, Modeposited substrates were loaded in the centre of a three-zone furnace (MTI model OTF-1200X) consisting precise temperature and gas flow controller units. The sulphur precursor purchased from Sigma-Aldrich was placed in the upstream of the growing chamber where the maximum temperature reached to $200{ }^{\circ} \mathrm{C}$, above than the sulphur melting point. Before heating process, the chamber was evacuated to $5 \mathrm{mTorr}$ and then the argon (Ar) gas was purged through the chamber to force undesired gases out. Then, the centre of the furnace was heated to $600{ }^{\circ} \mathrm{C}$ in $30 \mathrm{~min}$ and kept constant for next $15 \mathrm{~min}$. During this growth process, Ar gas was continuously flown (200 SCCM) as a carrier gas. Finally, growth chamber was cooled down to ambient temperature under the protection of Ar gas flow and samples were taken out for further experiments. Physical and electrochemical characteristics of vertically aligned $\mathrm{MoS}_{2}$ were characterized as previously discussed.

Density functional theory calculation. We have performed spin-polarized DFT calculations of $\mathrm{MoS}_{2}$ using SIESTA 3.1 (ref. 41) with the Perdew-Burke-Ernzerh of exchange-correlation functional ${ }^{42}$ and the norm-conserving Troullier-Martins pseudopotentials ${ }^{43}$ to describe valence electrons. The calculations were performed on a real-space grid with a mesh cutoff of $400 \mathrm{Ry}$ within the eigenvalue tolerance of $10^{-4} \mathrm{eV}$, using a DZP (double-zeta basis and polarization orbitals) basis set. The Brillouin zones of the unit cells were sampled by the Monkhorst-Pack grid with a spacing between k-points of $\Delta k<0.01 \AA^{-1}$. The geometry optimization was carried out within the conjugated gradient algorithm, until all the forces are $F<0.04 \mathrm{eV}$ per $\AA$ and the stress in the periodic direction is $\sigma<0.01 \mathrm{GPa}$. Quantum molecular dynamics simulations were performed using TeraChem ${ }^{44}$. The energies and forces were evaluated using the B3LYP exchange-correlation functional with $3-21 \mathrm{~g}$ basis set with DFT-D dispersion corrections ${ }^{45,46}$. The charges were calculated within the Mulliken scheme.

\section{References}

1. Keeling, R. \& Tans, P. NOAA/ESRL Trends in Atmospheric Carbon Dioxide, www.esrl.noaa.gov/gmd/ccgg/trends/.

2. Davis, S. J., Caldeira, K. \& Matthews, H. D. Future $\mathrm{CO}_{2}$ emissions and climate change from existing energy infrastructure. Science 329, 1330-1333 (2010).

3. Brook, E. J. Leads and lags at the end of the last Ice Age. Science 339, 1042-1043 (2013).

4. Parrenin, F. et al. Synchronous change of atmospheric $\mathrm{CO}_{2}$ and antarctic temperature during the last deglacial warming. Science 339, 1060-1063 (2013)

5. Whipple, D. T. \& Kenis, P. J. A. Prospects of $\mathrm{CO}_{2}$ utilization via direct heterogeneous electrochemical reduction. J. Phys. Chem. Lett. 1, 3451-3458 (2010).

6. Kumar, B. et al. Renewable and metal-free carbon nanofibre catalysts for carbon dioxide reduction. Nat. Commun. 4, 2819 (2013).

7. Rosen, B. A. et al. Ionic liquid-mediated selective conversion of $\mathrm{CO}_{2}$ to $\mathrm{CO}$ at low overpotentials. Science 334, 643-644 (2011).

8. Rosen, B. A. et al. In situ spectroscopic examination of a low overpotential pathway for carbon dioxide conversion to carbon monoxide. J. Phys. Chem. C 116, 15307-15312 (2012) 
9. Salehi-Khojin, A. et al. Nanoparticle silver catalysts that show enhanced activity for carbon dioxide electrolysis. J. Phys. Chem. C 117, 1627-1632 (2013).

10. Chen, Y., Li, C. W. \& Kanan, M. W. Aqueous $\mathrm{CO}_{2}$ reduction at very low overpotential on oxide-derived au nanoparticles. J. Am. Chem. Soc. 134, 19969-19972 (2012)

11. Li, C. W. \& Kanan, M. W. $\mathrm{CO}_{2}$ reduction at low overpotential on $\mathrm{Cu}$ electrodes resulting from the reduction of thick $\mathrm{Cu}_{2} \mathrm{O}$ films. J. Am. Chem. Soc. 134, 7231-7234 (2012).

12. Hoshi, N. Electrochemical reduction of $\mathrm{CO}_{2}$ on single crystal electrodes of silver $\operatorname{Ag}(111), \operatorname{Ag}(100)$ and $\operatorname{Ag}(110)$. J. Electroanal. Chem. 440, 283-286 (1997).

13. Rosen, B. A., Zhu, W., Kaul, G., Salehi-Khojin, A. \& Masel, R. I. Water enhancement of $\mathrm{CO}_{2}$ conversion on silver in 1-ethyl-3-methylimidazolium tetrafluoroborate. J. Electrochem. Soc. 160, H138-H141 (2013).

14. Lu, Q. et al. A selective and efficient electrocatalyst for carbon dioxide reduction. Nat. Commun. 5, 3242 (2014).

15. Chianelli, R. R. et al. Catalytic properties of single layers of transition metal sulfide catalytic materials. Catal. Rev. 48, 1-41 (2006).

16. Jaramillo, T. F. et al. Identification of active edge sites for electrochemical $\mathrm{H}_{2}$ evolution from $\mathrm{MoS}_{2}$ nanocatalysts. Science 317, 100-102 (2007).

17. Wang, T. et al. Size-dependent enhancement of electrocatalytic oxygenreduction and hydrogen-evolution performance of $\mathrm{MoS}_{2}$ particles. Chem. Eur. J. 19, 11939-11948 (2013).

18. Karunadasa, H. I. et al. A molecular $\mathrm{MoS}_{2}$ edge site mimic for catalytic hydrogen generation. Science 335, 698-702 (2012).

19. Hinnemann, B. et al. Biomimetic hydrogen evolution: $\mathrm{MoS}_{2}$ nanoparticles as catalyst for hydrogen evolution. J. Am. Chem. Soc. 127, 5308-5309 (2005).

20. Vayenas, C. G., Bebelis, S. \& Ladas, S. Dependence of catalytic rates on catalyst work function. Nature 343, 625-627 (1990).

21. Wang, Q. H., Kalantar-Zadeh, K., Kis, A., Coleman, J. N. \& Strano, M. S. Electronics and optoelectronics of two-dimensional transition metal dichalcogenides. Nat. Nano. 7, 699-712 (2012).

22. Chhowalla, M. et al. The chemistry of two-dimensional layered transition metal dichalcogenide nanosheets. Nat. Chem. 5, 263-275 (2013).

23. Bonde, J., Moses, P. G., Jaramillo, T. F., Nørskov, J. K. \& Chorkendorff, I Hydrogen evolution on nano-particulate transition metal sulfides. Faraday Discuss. 140, 219-231 (2009).

24. Zhou, W. et al. Intrinsic structural defects in monolayer molybdenum disulfide. Nano Lett. 13, 2615-2622 (2013).

25. Kibsgaard, J., Chen, Z., Reinecke, B. N. \& Jaramillo, T. F. Engineering the surface structure of $\mathrm{MoS}_{2}$ to preferentially expose active edge sites for electrocatalysis. Nat. Mat. 11, 963-969 (2012).

26. Li, Y. et al. $\mathrm{MoS}_{2}$ Nanoparticles grown on graphene: an advanced catalyst for the hydrogen evolution reaction. J. Am. Chem. Soc. 133, 7296-7299 (2011).

27. DuBois, D. L. in Encyclopedia of Electrochemistry. (ed Bard, A. J.) (Wiley-VCH Verlag GmbH \& Co. KGaA, 2007).

28. Łukaszewski, M., Siwek, H. \& Czerwiński, A. Electrosorption of carbon dioxide on platinum group metals and alloys-a review. J. Solid State Electrochem. 13, 813-827 (2008)

29. Gattrell, M., Gupta, N. \& Co, A. A review of the aqueous electrochemical reduction of $\mathrm{CO}_{2}$ to hydrocarbons at copper. J. Electroanal. Chem. 594, 1-19 (2006).

30. Freire, M. G., Neves, C. M. S. S., Marrucho, I. M., Coutinho, J. A. P. \& Fernandes, A. M. Hydrolysis of tetrafluoroborate and hexafluorophosphate counter ions in imidazolium-based ionic liquids. J. Phys. Chem. A 114 3744-3749 (2010).

31. Wamser, C. A. Hydrolysis of fluoboric acid in aqueous solution. J. Am. Chem. Soc. 70, 1209-1215 (1948)

32. Edward, J. T. Molecular volumes and the Stokes-Einstein equation. J. Chem. Educ. 47, 261-270 (1970).

33. Mazarei, A. F. \& Sandall, O. C. Diffusion coefficients for helium, hydrogen, and carbon dioxide in water at $25^{\circ} \mathrm{C}$. AIChE J. 26, 154-157 (1980).

34. Bard, A. J. Electrochemical Methods: Fundamentals and Applications (Wiley, 2001).

35. Hammer, B. \& Nørskov, J. K. Theoretical surface science and catalysiscalculations and concepts. Adv. Catal. 45, 71-129 (2000).

36. Botello-Méndez, A. R., López-Urías, F., Terrones, M. \& Terrones, H. Metallic and ferromagnetic edges in molybdenum disulfide nanoribbons. Nanotechnology 20, 325703-325706 (2009).
37. Li, Y., Zhou, Z., Zhang, S. \& Chen, Z. $\mathrm{MoS}_{2}$ nanoribbons: high stability and unusual electronic and magnetic properties. J. Am. Chem. Soc. 130, 16739-16744 (2008).

38. Wolf-Gladrow, D. A. Total alkalinity: the explicit conservative expression and its application to biogeochemical processes. Mar. Chem. 106, 287-300 (2007).

39. Kong, D. et al. Synthesis of $\mathrm{MoS}_{2}$ and $\mathrm{MoSe}_{2}$ films with vertically aligned layers. Nano Lett. 13, 1341-1347 (2013).

40. Lee, C. et al. Anomalous lattice vibrations of single- and few-layer $\mathrm{MoS}_{2}$. ACS Nano 4, 2695-2700 (2010).

41. Sanchez-Portal, D., Ordejon, P., Artacho, E. \& Soler, J. M. Density-functional method for very large systems with LCAO basis sets. Int. J. Quantum Chem. 65, 453-461 (1997).

42. Perdew, J., Burke, K. \& Wang, Y. Generalized gradient approximation for the exchange-correlation hole of a many-electron system. Phys. Rev. B 54, 16533-16539 (1996).

43. Troullier, N. \& Martins, J. Efficient pseudopotentials for plane-wave calculations. II. Operators for fast iterative diagonalization. Phys. Rev. B 43, 8861-8869 (1991).

44. Ufimtsev, I. S. \& Martinez, T. J. Quantum chemistry on graphical processing units. 3. analytical energy gradients, geometry optimization, and first principles molecular dynamics. J. Chem. Theory Comput. 5, 2619-2628 (2009).

45. Grimme, S., Antony, J., Ehrlich, S. \& Krieg, H. A consistent and accurate $a b$ initio parametrization of density functional dispersion correction (DFT-D) for the 94 elements H-Pu. J. Chem. Phys. 132, 154104 (2010).

46. Grimme, S., Ehrlich, S. \& Goerigk, L. Effect of the damping function in dispersion corrected density functional theory. J. Comput. Chem. 32, 1456-1465 (2011).

\section{Acknowledgements}

A.S.-K. work was supported by University of Illinois at Chicago through the Start-up and 'Proof of Concept' UIC Chancellor Award. P.K. work was supported by the ACS PRF grant \#53062-ND6. A.B. acknowledges the generous support obtained from the Herbert E. Paaren Graduate Fellowship. This work was carried out in part in the Frederick Seitz Materials Research Laboratory Central Facilities, University of Illinois. The acquisition of the UIC JEOL JEM-ARM200CF is supported by a MRI- ${ }^{2}$ grant from the National Science Foundation (DMR-0959470). This research used resources of the National Energy Research Scientific Computing Center (NERSC), which is supported by the Office of Science of the U.S. Department of Energy under Contract No. DE-AC02-05CH11231 and computational resources of the Extreme Science and Engineering Discovery Environment (XSEDE), which is supported by National Science Foundation grant No. OCI1053575.

\section{Author contributions}

A.S.-K., M.A. and B.K. conceived the idea. M.A., B.K., A.Be. and D.P. performed the electrochemical experiments, chemical vapour deposition growth process and characterizations, and A.S.-K supervised their efforts. R.F.K. and P.P. performed STEM measurements and analyses. P.K., A.Ba. and N.R. carried out DFT calculations. A.S.-K. and J.A. supervised M.A.'s efforts. R.H. performed UPS and XPS measurements B.A.R. and W.Z took some experiments to verify the results and participate in the discussion of electrochemical results. All of the authors contributed to the manuscript before submission.

\section{Additional information}

Supplementary Information accompanies this paper at http://www.nature.com/ naturecommunications

Competing Financial Interests: A.S.-K., M.A., B.K. filed a Provisional Patent Application, U.S. Patent Identification No. DG107, Filed February 2013. The other authors declare no competing financial interests.

Reprints and permission information is available online at http://npg.nature.com/ reprintsandpermissions/

How to cite this article: Asadi, M. et al. Robust carbon dioxide reduction on molybdenum disulphide edges. Nat. Commun. 5:4470 doi: 10.1038/ncomms5470 (2014) 